\title{
Approaching the linguistic complexity
}

\author{
Stanisław Drożdż ${ }^{1,2}$, Jarosław Kwapień ${ }^{1}$, and Adam Orczyk ${ }^{1}$ \\ 1 Polish Academy of Science, Institute of Nuclear Physics, 31-342 Kraków, Poland, \\ Stanislaw.Drozdz@ifj.edu.pl \\ 2 University of Rzeszów, Faculty of Mathematics and Natural Sciences, 35-310 \\ Rzeszów, Poland
}

\begin{abstract}
We analyze the rank-frequency distributions of words in selected English and Polish texts. We compare scaling properties of these distributions in both languages. We also study a few small corpora of Polish literary texts and find that for a corpus consisting of texts written by different authors the basic scaling regime is broken more strongly than in the case of comparable corpus consisting of texts written by the same author. Similarly, for a corpus consisting of texts translated into Polish from other languages the scaling regime is broken more strongly than for a comparable corpus of native Polish texts. Moreover, based on the British National Corpus, we consider the rank-frequency distributions of the grammatically basic forms of words (lemmas) tagged with their proper part of speech. We find that these distributions do not scale if each part of speech is analyzed separately. The only part of speech that independently develops a trace of scaling is verbs.
\end{abstract}

Key words: Complexity, natural language, Zipf law, word classes

\section{Introduction}

Even though central to the contemporary science the concept of complexity by its very nature - still leaves its precise definition a somewhat open issue. In intuitive and qualitative terms this concept refers to diversity of forms, to emergence of coherent and orderly patterns out of randomness, but also to a significant flexibility that allows switching among such patterns on a way towards searching for the ones that are optimal in relation to environment. Physics offers the methodology and concepts that seem promising for formalizing complexity. One of those concepts is criticality implying a lack of characteristic scale which indeed finds evidence in abundance of power laws and fractals in Nature.

Whatever definition of complexity one however adopts, the human language deserves a special status in the related investigations. It not only led humans to develop civilization but it also constitutes - from a scientific perspective - an extremely fascinating and complex dynamical structure [1]. Like many natural systems, language during its evolution developed remarkable complex patterns of behaviour such as hierarchical structure, syntactic organization, long-range correlations, and - what is particularly relevant here - a lack of characteristic scale. This latter phenomenon - in quantitative linguistics commonly referred 
to as the Zipf law - describes the rank-frequency distribution of words in a (sufficiently large) piece of text. This well-known, quantitatively formulated in 1949 by G.K. Zipf observation [2], based originally on "Ulysses" and latter on confirmed for many other literary texts, states that frequency of the rank-ordered words is inversely proportional to the words' rank. It needs to be added here that this law constitutes a principal reference in quantitative linguistics and inspiration for ideas and development in many different areas of science.

Zipf suggested interpretation of this law in terms of the so-called principle of least-effort $[2,5]$. This interpretation was however soon questioned after it had been shown that the Zipfian relation applies also to a "typewriting monkey" example [6], i.e. an essentially purely random process. This pointed to the Zipf law as too indiscriminate to reflect the complex organization of languages.

\section{Results and discussion}

Our recent studies based on English as well as on Polish texts open a new perspective to comprehend the linguistic complexity and sheds a new light on an involved message encoded in the Zipf law. First, referring to the classic analysis of "Ulysses" by James Joyce, carried out by G.K. Zipf [2], we compare properties of the rank-frequency distributions of words in the English and the Polish text [7] of this novel. Results are shown in Figure 1(a) and Figure 1(b). Both versions of the text show scaling behaviour over three decades between the ranks 10 and 10,000. However, the scaling exponent for the Polish text $(\alpha \simeq 0.90)$ is significantly smaller than for the English original $(\alpha \simeq 1.05)$. This difference might originate from a far more inflectable character of the Polish language, which demands a larger set of words (understood as particular sequences of letters) to reproduce the course of narration in "Ulysses". This observation may be considered a regularity, since typical Polish texts have smaller $\alpha$ than typical English texts. There are, however, Polish texts which have a value of $\alpha$ that is similar to English standards as it is documented in Figure 1(c). It is noteworthy that each of the three texts are well approximated by power-law distributions almost over the whole range of ranks.

Any text, as a piece of human syntactic communication, is not a series of grammatically equivalent words, but rather a convoluted mixture of words belonging to different parts of speech (classes). Tagging all the words with their proper parts of speech allows us to compare statistical properties of words within each class separately. Figure 2 shows the rank-frequency distributions of the most frequent nouns, verbs, adjectives, and adverbs in the British National Corpus [3] which is a representative sample of the contemporary written and spoken English. The data comes from [4]. As it can be seen, despite the fact that the whole corpus exhibits a Zipf-type scaling for ranks up to several thousands [9], the corresponding distributions may not necessarily show any scaling if only words representing a single part of speech are considered. Verbs are the only class of words that develop some trace of scaling behaviour with the scaling index $\alpha \approx 1$. 
Looking from this perspective at the global distribution of all the words belonging to all the classes together it is extremely interesting to reiterate that the entire rank-frequency distribution is Zipf-like.

This result can reflect a highly convoluted syntactic organization of human language, which may be considered as a complex system. From this angle, the linguistic complexity primarily manifests itself through the logic of mutual dressing among words belonging to different parts of speech that the entire proportions emerge scale-free even though in majority of these parts separately the proportions do not respect such a kind of organization. Another interesting issue open for speculation is whether the above results actually reflect the fact that the Zipf's principle of least action is more applicable to verbs - a part of speech related to action - than to nouns that are linked to objects. Worth considering is also a possibility that this reflects mapping of the well-established physical principle of least action onto the frequency distribution of verbs in a text.

As it was already mentioned above, passing from a single English text to a larger literary corpora is associated with reaching the limits of the applicability of the Zipf law in its classical form with the scaling index $\alpha=1$. Typically, after a short transient, for ranks larger than a few thousands another scaling regime is observed with $\alpha>1[8,9]$. Presence of two distinct scaling exponents in the rank-frequency distribution of English words can be explained by the existence of two sets of words: the first one comprises common words which are frequently used by all the authors (thus forming the language core), and the second one comprises the remaining words among which are technical words, words typical for a specific author or words which are otherwise rarely used. However, we propose another complimentary explanation of the breaking of the Zipf law for higher word ranks. Based on books of a few different authors we observe that the Zipf law is better realized for single texts than it is for corpora, even if we consider a corpus to be a collection of works of the same author. Figure 3 shows the rank-frequency distributions of words for two small corpora of Polish texts: the first one (black symbols) comprises 26 novels and stories by Polish fantasy writer Andrzej Sapkowski, while the second one (green symbols) is formed of 41 novels and stories written by 8 different authors. The texts in the second corpus were selected in such a way that the total length of each corpus is comparable (1.3 million words). It can easily be noted that for ranks larger that $6,000-8,000$ the distribution for the second corpus shows a slightly faster decay than the distribution for the first corpus. In turn, the distribution for the first corpus seems to deviate from the unique scaling behaviour more than any single member text of this corpus (not shown here, but compare this with the result for a single novel in Figure 1(c)). This conclusion, however, must be treated with care since single texts have much smaller vocabulary than larger corpora.

The above evidence suggests that the long-range correlations originating from a given book's continuous narration can be a strong source of scaling behaviour. These correlations are distorted if we form a corpus consisting of different works, in the same manner as the correlations which are allowed to exist in each particular realization of a system are suppressed if one forms a statistical ensemble 
from a number of different realizations of this system. This possibility opens space for contesting the traditional model of analysis in quantitative linguistics, according to which the corpora, due to their larger size, are more useful subject of analysis than single works. In our opinion this leads to losing a significant amount of information. The above outcome is another argument in favour of the concept that the words extracted from their context are rather different objects even from a purely statistical point of view than the same words embedded in a contextual environment.

Finally, let us look once again at Figure 1(a) and Figure 1(b), where the Zipf plots for an English text and its Polish translation are presented. Both distributions show the undistorted power-law slope for the whole range of ranks, which means that the scale-free character of "Ulysses" was preserved by the translator. Actually, if one takes into account the peculiar character of this novel, especially the unequally rich vocabulary, this result has to be considered remarkable. Motivated by this observation, we more systematically look at the rank-frequency distributions of words from texts which were translated into Polish from other languages. We find that although such texts show scale-free behaviour for the smallest ranks, for larger ones a breakdown of scaling occurs and we see a deflection towards smaller frequencies. In order to compare scaling properties of the translated and the native Polish texts, we constructed the following two small corpora: the first one consisting of 45 texts written originally in Polish, and the second one consisting of 30 translated texts. Both corpora have similar size of 2.3 million words. The results are presented in Figure 4. For the ranks $<2000$ both corpora develop roughly the same distributions with $\alpha \simeq 0.94$. However, for higher ranks the translated corpus is represented by less frequent words than the native corpus. The observed difference is sufficiently significant to consider it as an actual property of both considered groups of texts. This result does not seem to be unexpected. Generally, the higher is $\alpha$ for a sample, the poorer is the vocabulary of a corresponding author. It seems natural to expect that vocabulary of a writer is richer than a vocabulary of a translator. The first one works under no lexical constraints, while the second one has to concentrate principally on preserving the sense and the style of the original, what can lead to impoverishment of the lexicon. Moreover, differences in grammar also may play a role here.

The famous statement of P.W. Anderson [10], "More is different", seems particularly adequate in relation to the human language, indeed.

\section{References}

1. M.A. Nowak, J.B. Plotkin, V.A.A. Jansen, The evolution of syntactic communication, Nature 404, 495-498 (2000)

2. G.K. Zipf, Human behavior and the principle of least effort, Addison-Wesley (Cambridge, 1949)

3. The British National Corpus website: http://www.natcorp.ox.ac.uk/

4. G. Leech, P. Rayson, A. Wilson, Word Frequencies in Written and Spoken English: based on the British National Corpus, Longman (London 2001) 
5. R. Ferrer Cancho, R.V. Solé, Least effort and the origins of scaling in human language. Proc. Natl. Acad. Sci. USA 100, 788-791 (2003)

6. G.A. Miller, Some effects of intermittent silence, Amer. J. Psychol. 70, 311-314 (1957)

7. J. Joyce, Ulisses, translated by M. Słomczyński, Wydawnictwo Pomorze (Bydgoszcz 1992)

8. M.A. Montemurro, Beyond the Zipf-Mandelbrot law in quantitative linguistics, Physica A 300, 567-578 (2001)

9. R. Ferrer Cancho, R.V. Solé, Two regimes in the frequency of words and the origins of complex lexicons: Zipf's law revisited, J. Quant. Linguistics 8, 165-173 (2001)

10. P.W. Anderson, More is different, Science 177, 393-396 (1972) 
(a)

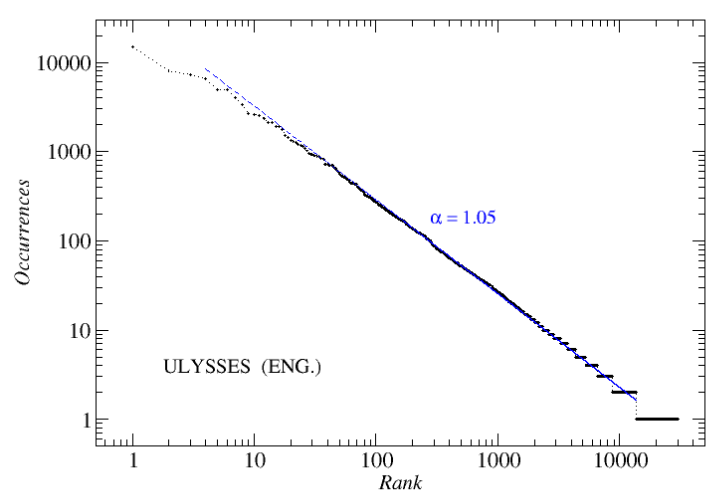

(b)

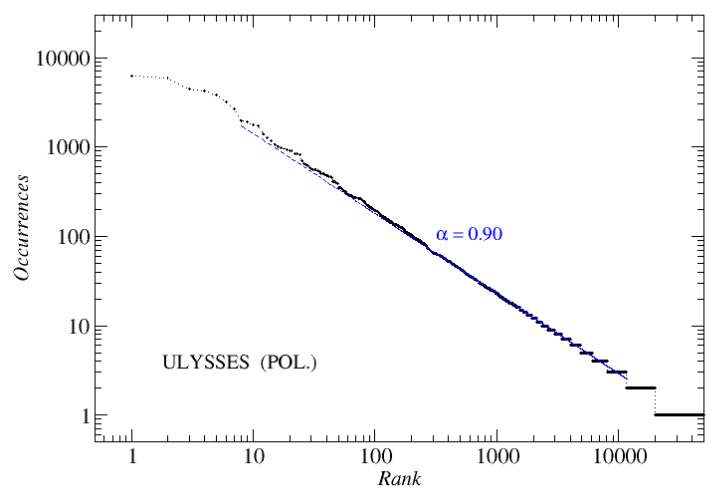

(c)

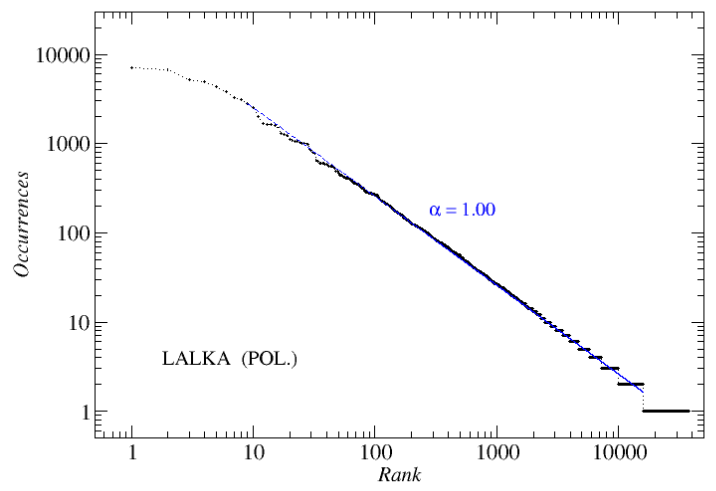

Fig. 1. Rank-frequency distributions of words in the English (a) and Polish (b) text of "Ulysses" by James Joyce, as well as in the native Polish text of "Lalka" by Bolesław Prus. All the three texts have comparable lengths of 240,000-260,000 words. 


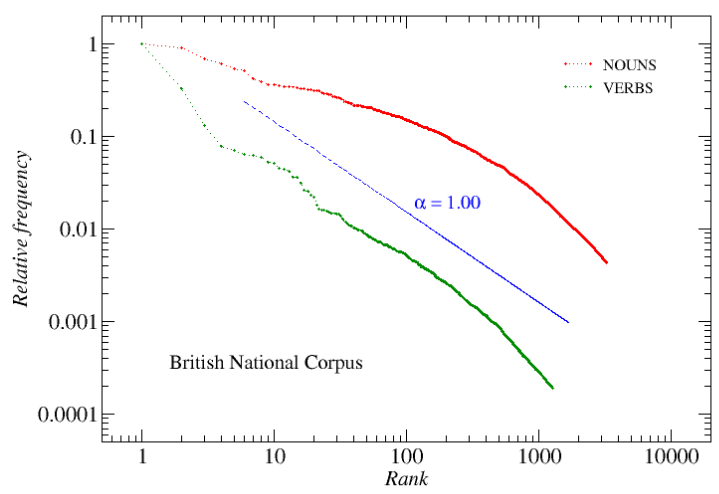

Fig. 2. Rank-frequency distributions of the most frequent lemmatized nouns (red) and verbs (green) taken from the British National Corpus. A slope with exponent $\alpha=1.00$ is shown as a benchmark.

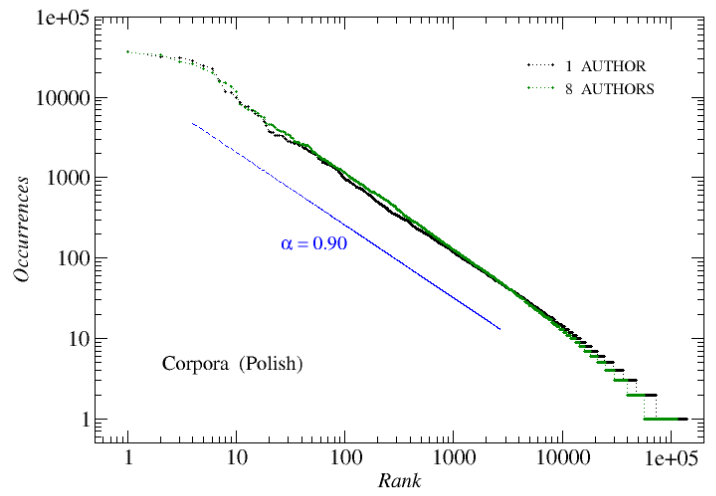

Fig. 3. Rank-frequency distributions of words for two Polish corpora consisting of works of the same author (black) and of different authors (green). A power-law with best-fitted exponent $\alpha \simeq 0.90$ is denoted by blue dashed line. 


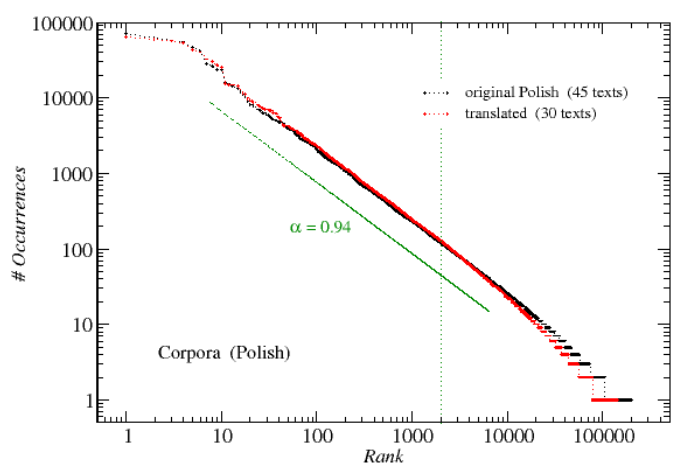

Fig. 4. Rank-frequency distributions of words for two Polish corpora consisting of 45 original texts written in Polish (black) and of 30 foreign texts translated into Polish (red). A power law with exponent $\alpha \simeq 0.94$ well-fitting both distributions within the ranks $10-2000$ is denoted by green dahed line. 